Article

\title{
Intensity of Radiative Recombination in the Germanium/Silicon Nanosystem with Germanium Quantum Dots
}

\author{
Sergey I. Pokutnyi ${ }^{1,2, *}$ and Lucjan Jacak ${ }^{3}$ \\ 1 Chuiko Institute of Surface Chemistry, National Academy of Sciences of Ukraine, 17 General Naumov Str., \\ 03164 Kyiv, Ukraine \\ 2 Institute of Physics, National Academy of Sciences of Ukraine, 46 Nauky Ave., 03028 Kyiv, Ukraine \\ 3 National Laboratory for Quantum Technologies, Wrocław University of Science and Technology, \\ 50-370 Wroclaw, Poland; Lucjan.Jacak@pwr.edu.pl \\ * Correspondence: isc-sec@i12.com
}

Citation: Pokutnyi, S.I.; Jacak, L.

Intensity of Radiative Recombination in the Germanium/Silicon

Nanosystem with Germanium Quantum Dots. Crystals 2021, 11, 275. https://doi.org/10.3390/cryst 11030275

Academic Editor: Paul J. Simmonds

Received: 24 February 2021

Accepted: 9 March 2021

Published: 11 March 2021

Publisher's Note: MDPI stays neutral with regard to jurisdictional claims in published maps and institutional affiliations.

Copyright: (c) 2021 by the authors. Licensee MDPI, Basel, Switzerland. This article is an open access article distributed under the terms and conditions of the Creative Commons Attribution (CC BY) license (https:// creativecommons.org/licenses/by/ $4.0 /)$.

\begin{abstract}
It is shown that in a germanium/silicon nanosystem with germanium quantum dots, the hole leaving the germanium quantum dot causes the appearance of the hole energy level in the bandgap energy in a silicon matrix. The dependences of the energies of the ground state of a hole and an electron are obtained as well as spatially indirect excitons on the radius of the germanium quantum dot and on the depth of the potential well for holes in the germanium quantum dot. It is found that as a result of a direct electron transition in real space between the electron level that is located in the conduction band of the silicon matrix and the hole level located in the bandgap of the silicon matrix, the radiative recombination intensity in the germanium/silicon nanosystem with germanium quantum dots increases significantly.
\end{abstract}

Keywords: spatially indirect excitons; hole tunneling; electron transition; quantum dots

\section{Introduction}

At present, research is being carried out aimed at increasing the efficiency of the intensity of radiative recombination and processes of interband light absorption in germanium/silicon heterostructures of the second type with germanium quantum dots (QDs). A solution to this problem would make it possible to create devices for optoelectronics and modern microelectronics on a single silicon substrate [1-8].

In heterostructures of the second type germanium/silicon with germanium QDs, electrons and holes are separated by a heterointerface. The holes are located in the valence band of the germanium QD, and the electrons are in the conduction band of the silicon matrix [9-21]. The spatial separation of electrons and holes, as a result of which electrons were localized above the QD and holes moved into QD, was found in the germanium/silicon heterostructure [9-21]. Such excited states have been called spatially indirect excitons (SIE) [9-22]. Interband transitions between electron and hole states in such a nanosystem are indirect in space. A consequence of the spatial separation of electrons and holes in the germanium/silicon heterostructure with germanium QDs was the small value of the overlap integral of the electron and hole wave functions (about a few hundredths). Since the intensity of optical transitions in the nanosystem was determined by the square of the overlap integral of the electron and hole wave functions, then in such a heterostructure, the experimentally observed weak intensity of optical transitions [1].

The theoretical description of SIE, comprising of an interacting mobile hole in a germanium QD with a localized electron over the spherical QD/matrix interface, was developed in [23-25]. The theory predicted a significant-almost two orders of magnitudeincrease of the SIE binding energy $E_{e x}(a)$, which was dependent on the QD radius $a$, against the exciton binding energy in a bulk crystalline silicon. The effect can be explained due to 
the centrifugal potential impact as well to polarization interaction one with the spherical QD/matrix interface one [23-25].

A theory of optical absorption via SIEs arising at the germanium QDs interface with silicon matrix was developed in [26]. It was found that the (dipole moments)/(oscillator strengths) of the transitions and the transient polarizability of SIE states considerably exceed the corresponding ones of conventional semiconductors under low-intensity light irradiation [26].

It was shown that electron tunneling through a potential barrier separating two germanium QDs caused the splitting of electron states localized over spherical QD/matrix interfaces. As a result, the mentioned splitting gave rise to a band of localized electron states, being in the band gap of the silicon matrix [27].

In the experimental work [2], the photoluminescence of germanium/silicon heterostructures with double vertically coupled germanium QDs was investigated. The germanium QDs were pyramidal with a larger base side of $40 \mathrm{~nm}$. The size of the short side of the base was 10-15 nm, and the height was 1-1.5 $\mathrm{nm}$. The germanium nanoclusters were separated by a silicon layer $d=30 \mathrm{~nm}$ thick. In [2], broad bands were observed in the range (720-820 meV) of the photoluminescence spectra. These bands in the photoluminescence spectra in [2] were caused by optical transitions between hole states in the germanium QDs and electronic states in the silicon layer localized near the QDs.

The results obtained in [2] confirmed the theoretical predictions [1], in which the electron states localized near the edges of the bases of germanium nanoclusters had a high probability of electron penetration into QDs. The strong tunneling coupling between QDs increased the probability of finding a hole at the edge of the germanium nanocluster. This caused an increase in the radiative recombination intensity in the heterostructure.

In experimental works [5,6], the photoluminescence of combined Ge/Si heterostructures was investigated. These heterostructures were a combination of disk-shaped Si/Ge QDs with sizes (200-250 nm) and groups of laterally ordered QDs grown in the deformation field of nanodisks. The sizes of groups of laterally ordered QDs were 40-50 nm. It was found that a multiple increase in the photoluminescence intensity of heterostructures was caused by a change in the parameters of vertically oriented QD groups. It was found that the arrangement of QDs in compact groups determined the effective localization of an electron with an almost equal probability of finding an electron in the silicon matrix and in the barrier of germanium QDs. In this case, the photoluminescence of the heterostructures at room temperature was caused by the accumulation of deformation in the multilayer structure of laterally ordered QDs.

In experimental works $[7,8]$, it was found that the use of ion irradiation in the process of epitaxial growth leads to a multiple increase in the photoluminescence of Ge/Si QDs. It was suggested that the enhancement of the photoluminescence of the heterostructure occurred due to the introduction of deep centers through which the process of radiative recombination proceeds.

In [28], a variational method was developed for finding the energy of the ground state of an electron and a hole in pyramidal germanium QDs grown in a silicon matrix. In this case, the SIE Hamiltonian lacked the electron-hole Coulomb interaction energy. In addition, in [29], the possibility of a hole leaving the germanium QD into the silicon matrix was not taken into account.

Theoretical studies of optical transitions in a nanosystem consisting of germanium QDs grown in a silicon matrix are currently underdeveloped. In particular, there is no SIE theory in a nanosystem that would take into account a hole leaving the germanium QD into the silicon matrix. To fill a gap in the theory, in this work, we develop the theory of SIE in a nanosystem taking into account the possibility of a hole leaving the valence band of germanium QDs into the bandgap of the silicon matrix. In this case, the SIE Hamiltonian contains the electron-hole Coulomb interaction energy. In order to obtain analytical expressions for the energies of the ground state of an electron, hole, and SIE, as well as for the radiative recombination intensity, in this work, we consider a model of 
a nanosystem consisting of a spherical germanium QD. A direct transition in real space is possible between an electron located in the conduction band of the silicon matrix and a hole located in the bandgap of the silicon matrix. It is shown that as a result of such a direct electron transition in real space, the intensity of radiative recombination in the germanium/silicon nanosystem with germanium QDs increases significantly.

\section{Energies of Electron and Hole States in a Nanosystem}

In germanium/silicon heterostructures with germanium QDs of the second type, the main electron level was in the silicon matrix, and the main level of holes was in the germanium QD [9-15]. The localization of holes in the QD was due to a significant shift of the upper edge of the valence band $\left(\Delta E_{v(G e)}=700 \mathrm{meV}\right)$ of germanium QD (relative to the upper edge of the valence band of the silicon matrix) [9-15]. In the heterostructure, the electrons moved in the silicon matrix and did not penetrate into the germanium QDs, since a substantial shift of the bottom of the conduction band $\left(\Delta E_{c(S i)}=340 \mathrm{meV}\right)$ of the silicon matrix (relative to the bottom of the conduction band of germanium QDs) was observed on the interface (a QD-silicon matrix). In this case, the electron-hole Coulomb interaction energy causes localization of the electron in a potential well above the surface of the QD. The appearance of SIE was found in the germanium/silicon heterostructure [9-15].

Consider a model of a quasi-zero-dimensional nanosystem containing a spherical germanium QD with a radius a and permittivity $\varepsilon_{2}=16.3$, in silicon matrix with $\varepsilon_{1}=11.7$. In this nanosystem, a hole with effective mass $\left(\left(m_{h, 2} / m_{0}\right)=0.39\right)$ moves in the QD volume, and an electron with effective mass $\left(\left(m_{e, 1} / m_{0}\right)=0.98\right)$ moves in the silicon matrix $\left(m_{0}\right.$ is free electron mass) [24,25].

Let us consider the SIE model, in which a hole with an effective mass $m_{h, 2}$ is in the valence band of a germanium QD, and an electron with an effective mass $m_{e, 1}$ is localized above the spherical surface of a QD, and it moves in the band conductivity of the silicon matrix. In the model of a quasi-zero-dimensional nanosystem in the framework of the effective-mass approximation, the Hamiltonian of the SIE takes the form [24,25].

$$
H\left(r_{e}, r_{h}, a\right)=-\left(\hbar^{2} / 2 m_{e, 1}\right) \Delta-\left(\hbar^{2} / 2 m_{h, 2}\right) \Delta+V_{e h}(r)+\left(E_{g(G e)}-\Delta E_{c(S i)}\right)
$$

where the first three terms are the operators of kinetic energy of the electron and hole, the value $\left(E_{g(G e)}-\Delta E_{c(S i)}\right)=330 \mathrm{meV}$, and $E_{g(G e)}$ is the bandgap energy of the germanium QD. The input from the polarizing interaction between the electron-hole and the QD surface, which is described using expressions obtained in $[5,6]$ can be neglected in the exciton Hamiltonian (1) (because the dielectric constants of the QD and the matrix are slightly different). In Hamiltonian (1), the electron-hole Coulomb interaction energy $V_{e h}(r)$ looks like [23-25]:

$$
V_{e h}(r)=-e^{2} / \widetilde{\varepsilon} r
$$

where $r=\left|r_{e}-r_{h}\right|, r_{e}$ and $r_{h}$ are electron and hole distances from the QD center, and $\widetilde{\varepsilon}=2 \varepsilon_{1} \varepsilon_{2} /\left(\varepsilon_{1}+\varepsilon_{2}\right)$ is the dielectric constant of the nanosystem.

Let us consider the case in which the average radii $a$ of the germanium QD in the nanosystem is much smaller than the Bohr radius $a_{h}$ of a hole in QD, i.e.,

$$
a \ll a_{h}
$$

(where $a_{h}=\varepsilon_{2}\left(m_{0} / m_{h, 2}\right)\left(\hbar^{2} / m_{0} e^{2}\right)$. In this case, the kinetic energy $\left(\approx\left(\hbar^{2} / 2 m_{h, 2} a^{2}\right)\right)$ of a hole in a QD significantly exceeds the electron-hole Coulomb interaction energy $V_{e h}(r)(2)$ :

$$
\left(\hbar^{2} / 2 m_{h, 2} a^{2}\right) \gg\left|V_{e h}(r)\right| \approx\left(e^{2} / \widetilde{\varepsilon} a\right)
$$

In germanium QDs with an average radius $a$ from interval (3), according to (4), the interaction of a hole with an electron can be neglected. In this case, the motion of a hole in 
a QD will be independent of the motion of an electron. Therefore, the Hamiltonian (1) of the SIE can be divided into two Hamiltonians. The first Hamiltonian,

$$
H_{h}\left(r_{h}\right)=-\left(\hbar^{2} / 2 m_{h, 2}\right) \Delta+E_{g(G e)},
$$

describes the motion of a hole in the germanium QD, and the second Hamiltonian,

$$
H_{e}\left(r_{e}, r_{h}, a\right)=-\left(\hbar^{2} / 2 m_{e, 1}\right) \Delta+V_{e h}(r)+E_{g(S i)},
$$

describes the motion of an electron localized above the interface (QD in the silicon matrix). In Equation (6), $E_{g(S i)}$ is the bandgap energy in a silicon matrix.

The energies of hole states in germanium QD are determined by the solution of the Schrödinger equation containing the Hamiltonian $H_{h}\left(r_{h}\right)(5)$. The energies of these hole states are defined as $[29,30]$ :

$$
E_{n, l}(a)=\left(\hbar^{2} / 2 m_{h, 2} a^{2}\right) \varphi_{n, l^{2}}
$$

where the subscripts $(n, l)$ refer to the corresponding quantum size-confined states. Here, $n$ and $l$ are the principal and azimuthal quantum numbers for the hole, and $\varphi_{n, l}$ are the roots of the Bessel function, i.e., $J_{l+1 / 2}\left(\varphi_{n, l}\right)=0$. To ascertain the quantum-limited levels in QD, it is necessary that the hole energy $E_{n, l}(a)(7)$ be much larger than the electron-hole Coulomb interaction energy $V_{e h}(r)(2)$,

$$
E_{n, l}(a) \gg\left|V_{e h}(r)\right| \approx\left(e^{2} / \widetilde{\varepsilon} a\right) .
$$

Condition (8) is satisfied for QDs radii,

$$
a \ll\left(\varphi_{n, l}^{2} / 2\right)\left(\widetilde{\varepsilon} / \varepsilon_{2}\right) a_{h} .
$$

In a nanosystem containing a germanium QD with a mean radius $a$ satisfying condition (9), the hole motion will be independent of the electron motion. Therefore, the energy $E_{1,0}{ }^{(e x)}(a)$ of the SIE ground state in the nanosystem has the form:

$$
E_{1,0}{ }^{(e x)}(a)=E_{1,0}^{(e)}(a)+E_{(G e) 1,0}^{(h)}(a, \Delta V) .
$$

The energy $E_{1,0}{ }^{(e x)}(a)(10)$ of the SIE ground state consists of the energy $E_{1,0}{ }^{(e)}(a)$ of the ground state of an electron moving in silicon matrices and also with the energy $E_{(G e) 1,0}{ }^{(h)}(a, \Delta V)$ of the ground state of a hole located in the germanium QD. The hole energy $E_{(G e) 1,0}{ }^{(h)}(a, \Delta V)$ will be found taking into account the possibility of a hole, leaving the valence band of germanium QDs into the bandgap of the silicon matrix.

The results of the variational calculation of the energy,

$$
E_{1,0}{ }^{(e)}(a, j(a))=\left\langle\Psi(r)\left|H_{e}(r, a)\right| \Psi(r)\right\rangle,
$$

of the ground state of an electron are obtained for the nanosystem containing germanium QDs grown in a silicon matrix with a mean QD radius $a$ in the interval,

$$
1.1 \mathrm{~nm} \leq a \leq 1.5 \mathrm{~nm} .
$$

In Equation (11), the Hamiltonian $H_{e}(r, a)$ of an electron is determined by expression (6). The values of the mean radius $a$ of the germanium QDs from interval (12) satisfy condition (9). We write the variational wave function of the ground state of an electron in a nanosystem in the form of an exciton-like wave function,

$$
\Psi_{1,0}(r)=A(r-a) \exp (-j(r-a) / a) .
$$


In (13), the normalized coefficient $A$ is determined by the expression,

$$
A=j^{5 / 2} / \pi^{1 / 2} a^{5 / 2}\left(j^{2}+3 j+3\right)^{1 / 2}
$$

where $j=j(a)$ is a variation parameter. The variational wave function of the ground state of an electron (13) is determined in the range of distances $a \leq r<\infty$. Since the electron cannot penetrate into the germanium $\mathrm{QD}$, then at the interface (QD is the matrix) at $r=a$, the electron wave function $\Psi_{1,0}(r=a)=0$. When obtaining the energy $E_{1,0}{ }^{(e)}(a, j(a))(11)$ of the ground state of an electron by the variational method, the electron-hole Coulomb interaction energy $V_{e h}(r)$ (2) was taken into account in Hamiltonian (6).

The germanium QD, in the valence band of which a hole with an effective mass $m_{h, 2}$ moves, will be modeled by a spherical potential well with a depth $\Delta V=\Delta E_{v(\mathrm{Ge})}=$ $700 \mathrm{meV}$. The energy $E_{(G e) 1,0}{ }^{(h)}(a, \Delta V)$ of the hole, taking into account the possibility of a hole emerging from the germanium QD into the silicon matrix, is obtained from the equation [29]:

$$
\tan (p a)=K(p a)
$$

where function,

$$
K(p a)=(p a)\left[1-\beta-\beta x\left[1-\left(\alpha^{2} / \beta\right)\right]^{1 / 2}\right]^{-1} .
$$

In (15) and (16), we introduce the following notation:

$$
\begin{gathered}
(p a)=(x \alpha)=\left[\left(2 m_{h, 2} a^{2} / \hbar^{2}\right) E_{(G e) 1,0}(h)(a, \Delta V)\right]^{1 / 2} \\
\beta=\left(m_{h, 2} / m_{h, 1}\right), x=\left[\left(2 m_{h, 2} a^{2} / \hbar^{2}\right)(\Delta V / \beta)\right]^{1 / 2}, \alpha=\left[\beta\left(E_{(G e) 1,0}(h)(a, \Delta V) / \Delta V\right)\right]^{1 / 2} .
\end{gathered}
$$

As a result of the exit of a hole with an effective mass $m_{h, 2}$ from the valence band of the germanium QD, a hole appears in the band gap of the silicon matrix at the energy level $E_{(S i) 1,0}{ }^{(h)}(a, \Delta V)$ with an effective mass of $\left(m_{h, 1} / m_{0}\right)=0.5$ (see Figure 1).

The energy value $E_{(S i) 1,0}{ }^{(h)}(a, \Delta V)=E_{(G e) 1,0}{ }^{(h)}(a, \Delta V)$ is determined from Equations (15)-(18). In this case, the hole is described by the wave function [29]:

$$
\Psi_{1,0}{ }^{(h)}(r)=B \exp (-\lambda r) / 2 \pi^{1 / 2} r .
$$

In (19), the normalized coefficient $B$ is determined by the expression,

$$
B=2 \sin (p a) \exp (\lambda a) / a^{1 / 2}\left[1-(\sin (2 p a) / 2 p a)+\left(\sin ^{2}(p a) / \lambda a\right)\right]^{1 / 2},
$$

where the parameter of the nanosystem,

$$
(\lambda a)=\beta^{-1}(p a)\left[\left(\Delta V / E_{1,0}(h)(a, \Delta V)\right)-1\right]^{1 / 2} .
$$

The hole wave function (19) is defined in the range of distances $a \leq r<\infty$.

In a germanium/silicon nanosystem with germanium QDs in the interval of average QD radii $a$ (6) upon absorption of a quantum of light with energy,

$$
\hbar \omega_{e h}(a)=E_{(G e) 1,0}{ }^{(h)}(a, \Delta V)+\left(E_{g(G e)}-\Delta E_{c(S i)}\right)+E_{1,0}{ }^{(e)}(a),
$$

the ground state of the SIE with the energy $E_{1,0}{ }^{(e x)}(a)(10)$ appeared. The energy level $E_{(G e) 1,0}{ }^{(h)}(a, \Delta V)$ of the hole is measured from the top of the QD valence band. The energy level $E_{1,0}(e)(a)$ (11) of an electron is measured from the bottom of the conduction band of the silicon matrix (see Figure 1). 

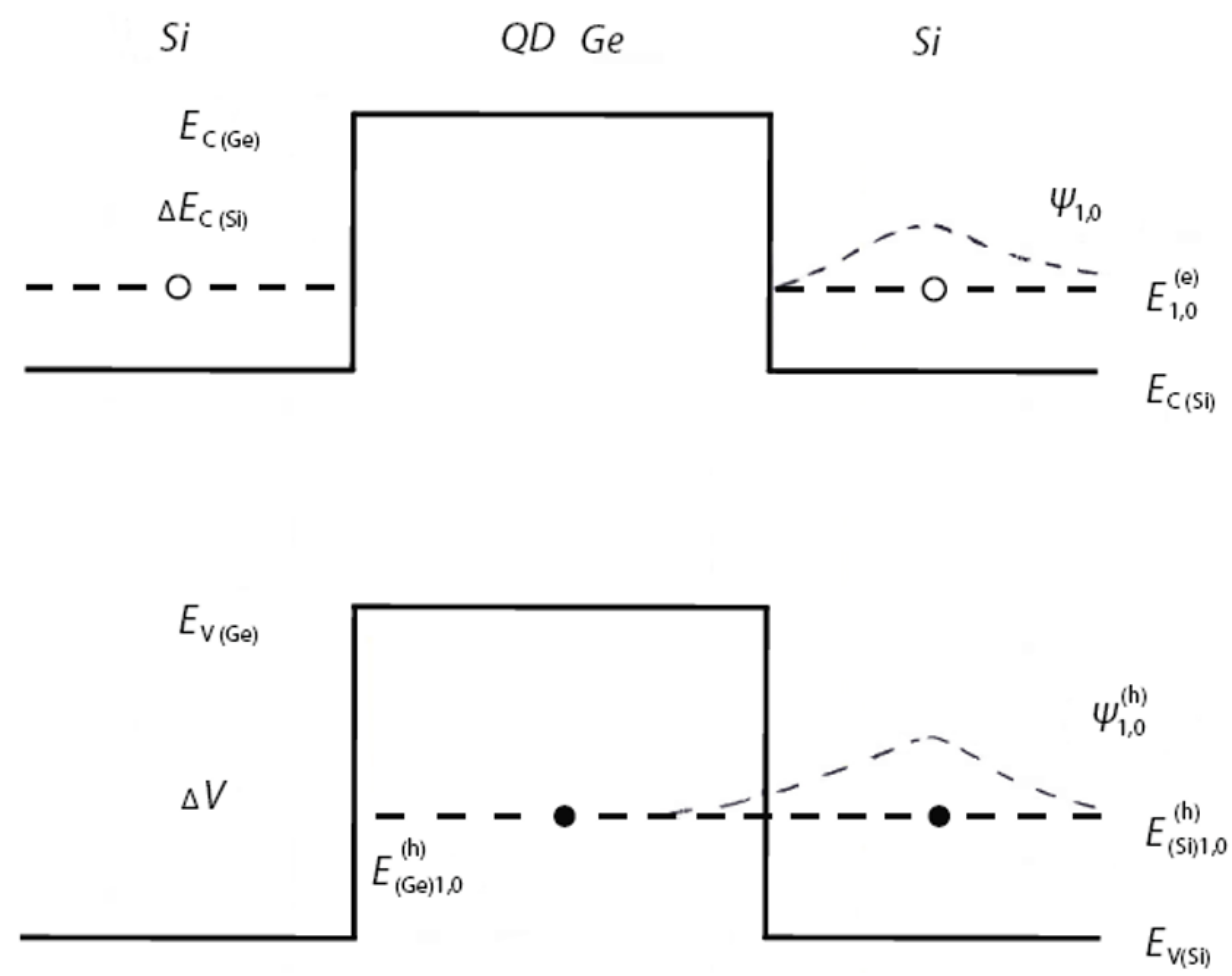

Figure 1. Band diagram of a nanoheterostructure containing a single germanium quantum dot (QD) grown in a silicon matrix. In the nanoheterostructure, the QD is a potential well for a hole (denoted by $\bullet$ ) and a potential barrier for an electron (denoted by $\bigcirc$ ). The energies $E_{\mathrm{c}(G e)}, E_{\mathrm{v}(G e)}$ and $E_{\mathrm{c}(S i)}$, $E_{\mathrm{v}(S i)}$ correspond to the positions of the bottom of the conduction band and the top of the valence band of the silicon matrix and germanium QD, respectively. $\Delta E_{\mathrm{c}(S i)}$ and $\Delta V$, respectively, represent the shift of the bottom of the conduction band in the silicon matrix relative to the bottom of the conduction band of germanium QDs and shift of the top of the valence band in the silicon matrix relative to the top of the valence band of the QD of germanium. $E_{1,0}{ }^{(e)}$ and $E_{(S i) 1,0}{ }^{(h)}, E_{(G e) 1,0}{ }^{(h)}$, respectively, represent the position of the level of an electron in the conduction band of the silicon matrix and the position of the level of a hole in the bandgap of the silicon matrix, and the position of the level of a hole in the valence band of the QD of germanium. The figure also shows (dotted line) the distributions of the wave function $\Psi_{1,0}$ of an electron located in the conduction band of the silicon matrix and the wave function $\Psi_{1,0}{ }^{(h)}$ of a hole located in the bandgap of the silicon matrix.

\section{Intensity of Optical Transitions in the Nanosystem}

The intensity $\left(I_{1,0}(a) / C\right)$ of the dipole-allowed transition of an electron in real space between the electron $E_{1,0}{ }^{(e)}(a)(11)$ and hole $E_{(S i) 1,0}^{(h)}(a, \Delta V)((15)-(18))$ energy levels, located in the conduction band and the bandgap of the silicon matrix, is determined by the square of the overlap integral of the electron $\Psi_{1,0}(r)(13),(14)$ and hole $\Psi_{1,0}{ }^{(h)}(r)(19),(20)$ wave functions [1]:

$$
I_{1,0}(a)=C\left|\int_{a}^{\infty} \Psi_{1,0}(r) \Psi_{1,0}{ }^{(h)}(r) r^{2} d r\right|^{2} \delta\left(\hbar \omega-\hbar \omega_{e h}(a)\right) .
$$

In (23), $C$ is the quantity proportional to the square of the modulus of the matrix element of the dipole moment, which is taken on the Bloch functions of the conduction and valence bands of germanium and silicon [1], $\omega$ is the frequency of the emitting light, and the radiation energy $\hbar \omega_{e h}(a)$ is determined by Formula (22). Integrating in (22), taking into account (13), (14) and (19), (20), we obtain an expression that describes the intensity of optical transition, 


$$
\begin{gathered}
I_{1,0}(a) / C=j^{5}(\lambda a+j+2)^{2} \sin ^{2}(p a) / \pi^{2}(\lambda a+j)^{6}\left(j^{2}+3 j+3\right) \times[1- \\
\left.(\sin (2 p a) / 2 p a)+\left(\sin ^{2}(p a) / \lambda a\right)\right] .
\end{gathered}
$$

The value of the square of the overlap integral of the electron $\Psi_{1,0}(r)(13),(14)$, and hole $\Psi_{1,0}{ }^{(h)}(r)(19)$, (20) wave functions, as well as the radiation intensity $\left(I_{1,0}(a) / C\right)(23)$, (24), depends on the possibility of a hole leaving the valence band of germanium QDs into the bandgap of the silicon matrix. The hole tunneling efficiency is determined by the position of the energy level $E_{(G e) 1,0}{ }^{(h)}(a, \Delta V)$ of the hole relative to the depth $\Delta V$ of the spherical potential well of the germanium QD. As the level $E_{(G e) 1,0}{ }^{(h)}(a, \Delta V)$ of the hole approaches the value of $\Delta V$, there is the possibility of a hole leaving the germanium QD into the bandgap of the silicon matrix. When a hole leaves the germanium $\mathrm{QD}$, a hole appears in the bandgap of the silicon matrix at the energy level $E_{(S i) 1,0}{ }^{(h)}(a, \Delta V)=E_{(G e) 1,0}(h)(a, \Delta V)$ ((15)-(18)) (see Figure 1). As a result, the average distance between an electron located at the energy level $E_{1,0}{ }^{(e)}(a)(11)$ in the conduction band of the silicon matrix and a hole at the energy level $E_{(S i) 1,0}(e)(a, \Delta V)((15)-(18))$ in the bandgap of the silicon matrix decreases significantly. This also leads to a significant increase in the square of the overlap integral of the electron $\Psi_{1,0}(r)(13),(14)$ and hole $\Psi_{1,0}{ }^{(h)}(r)(19),(20)$ wave functions. In this case, the condition is satisfied,

$$
D(a, \Delta V)=\left[\left(\Delta V / E_{1,0}{ }^{(h)}(a, \Delta V)\right)-1\right] \ll 1 .
$$

In the case of strong hole tunneling (25), the radiation intensity (24) caused by the transition of an electron in real space between energy levels ((15)-(18)), located in the conduction band and in the bandgap of the silicon matrix, takes on the importance of order,

$$
\left(I_{1,0}(a) / C\right) \sim(\lambda a)^{-4} \sim \beta^{4}(p a)^{-4}\left[\left(\Delta V / E_{1,0}(h)(a, \Delta V)\right)-1\right]^{-2} .
$$

\section{Numerical Results}

Table 1 shows the energy values $E_{1,0}{ }^{(e x)}(a)(10)$ of the SIE ground state, energy $E_{(G e) 1,0}{ }^{(h)}(a, \Delta V)$ of the hole ground state (obtained using Formulas (15)-(18)), energy $E_{1,0}(e)(a)$ (11) of the ground state of an electron (variation calculation), energy $\hbar \omega_{e h}(a)$ (22) of electron transition and radiation intensity $\left(I_{1,0}(a) / C\right)(24)$, as a function of the mean radius $a$ of the germanium QD, for a nanosystem containing germanium QDs grown in a silicon matrix, with mean QD radii $a$ in the interval (12). With an increase in the mean radius $a$ of germanium QDs from interval (12) from $a=1.1$ to $1.5 \mathrm{~nm}$, the energy values $E_{1,0}{ }^{(e x)}(a)$ (10) SIE significantly decrease from 744.2 to $406.4 \mathrm{meV}$ (see Table 1). There is a significant decrease in the values of the energy $E_{(G e) 1,0}(h)(a, \Delta V)$ of the hole from 658.8 to $346.4 \mathrm{meV}$ and the energy $E_{1,0}{ }^{(e)}(a)(11)$ of an electron from 85.4 to $60 \mathrm{meV}$, and also a significant decrease from 1074.2 to $736.4 \mathrm{meV}$ of the transition energies $\hbar \omega_{\text {eh }}(a)$ (18) with an increase in the mean radius $a$ of germanium QDs from $a=1.1$ to $1.5 \mathrm{~nm}$ (see Table 1). These decreases in energies $E_{1,0}{ }^{(e x)}(a)(10), E_{1,0}(e)(a)(11), E_{(G e) 1,0}{ }^{(h)}(a, \Delta V)$ $((15)-(18))$, and $\hbar \omega_{e h}(a)$ (22) with increasing radius $a$ of the $\mathrm{QD}$ are caused by the fact that in the interval (12), the radius $a \mathrm{QD}$, according to the effect of dimensional quantization of quasiparticles, the main contribution to the SIE energy and hole energy is made by the dependence $E_{1,0}{ }^{(e x)}(a), E_{1,0}(h)(a, \Delta V) \approx a^{-2}$. A decrease in the energy $E_{1,0}{ }^{(e)}(a)(11)$ of an electron with an increase in the mean radius $a$ of a germanium QD from $a=1.1$ to $1.5 \mathrm{~nm}$ is due to a decrease in the electron-hole Coulomb interaction energy $\left|V_{e h}(r)\right|(2)$ (in this case, $\left.\left|V_{e h}(r)\right| \approx a^{-1}\right)$. This decrease in the energy $E_{1,0}(e)(a)(11)$ of the electron is weaker expressed (in comparison with a decrease in energies $E_{1,0}{ }^{(e x)}(a)(10)$ and $E_{(G e) 1,0}{ }^{(h)}(a, \Delta V)$ $((15)-(18)))$, since, according to (2), the energy $E_{1,0}{ }^{(e)}(a) \approx a^{-1}$. 
Table 1. Dependences of energy $E_{1,0}{ }^{(e x)}(a)(10)$ of the spatially indirect excitons (SIE) ground state (variational calculation) (expressed by meV), energy $E_{(G e) 1,0}{ }^{(h)}(a, \Delta V)$ of a hole (expressed by meV) (obtained using Formulas (15)-(18)), and energy $E_{1,0}{ }^{(e)}(a)(11)$ of an electron (expressed by meV), energy $\hbar \omega_{e x}(a)$ (22) of an electron transition (expressed by meV), and radiation intensity $\left(I_{1,0}(a) / C\right)(24)$, as a function of the mean radius $a$ (expressed by nm) QD germanium. $\Delta V=700 \mathrm{meV}$ is the depth of the potential well for holes in the germanium QD.

\begin{tabular}{|c|c|c|c|c|c|c|}
\hline $\mathrm{a}, \mathrm{nm}$ & $E_{(G e) 1,0}^{(h)} \mathrm{meV}$ & $E_{1,0}{ }^{(e)}, \mathrm{meV}$ & $E_{1,0}{ }^{(e x)}, \mathrm{meV}$ & $\hbar \omega_{e h}, \mathrm{meV}$ & $E_{(G e) 1,0}{ }^{(h)} / \Delta V$ & $I_{1,0} / C\left(10^{-2}\right)$ \\
\hline 1.1 & 658.8 & 85.4 & 744.2 & 1074.2 & 0.94 & 22 \\
\hline 1.2 & 552 & 78.2 & 630.2 & 960.2 & 0.79 & 15 \\
\hline 1.3 & 468.4 & 71.8 & 540.2 & 870.2 & 0.67 & 10.4 \\
\hline 1.4 & 401.2 & 65.6 & 466.8 & 796.8 & 0.57 & 6.5 \\
\hline 1.5 & 346.4 & 60 & 406.4 & 736.4 & 0.5 & 3 \\
\hline
\end{tabular}

The radiation intensity $\left(I_{1,0}(a) / C\right)(24)$ caused by the transition of an electron in real space between the energy levels $E_{1,0}(e)(a)(11)$ and $E_{(S i) 1,0}(e)(a, \Delta V)((15)-(18))$, located in the conduction band and in the bandgap of the silicon matrix, with an increase in the mean radius $a$ of germanium QDs from $a=1.1$ to $1.5 \mathrm{~nm}$, significantly decreases from $22 \cdot 10^{-2}$ to $3 \cdot 10^{-2}$ (see Table 1 ). In the case of strong tunneling (25), (26) of a hole with a QD of radius $a=1.1 \mathrm{~nm}$ into the silicon matrix, the value $D=0.055$, and the radiation intensity $\left(I_{1,0}(a) / C\right)(24)$ takes on a significant value $22 \cdot 10^{-2}$. This value of the radiation intensity is more than an order of magnitude higher than the intensity $\left(\cong 10^{-2}\right)$ obtained in the experimental work [2]. With an increase in the mean radius $a$ of germanium QDs from $a=$ 1.1 to $1.5 \mathrm{~nm}$, the energy $E_{(\mathrm{Ge}) 1,0}{ }^{(h)}(a, \Delta V)$ of the hole ground state decreases, which leads to the distance of the level $E_{(G e) 1,0}{ }^{(h)}(a, \Delta V)$ of the hole from $\Delta V$ (see Table 1). This causes a decrease in the possibility of a hole emerging from the $\mathrm{QD}$ and also a significant decrease in the square of the overlap integral of the electron $\Psi_{1,0}(r)(13),(14)$ and hole $\Psi_{1,0}(h)(r)(19)$, (20) wave functions. As a result, the radiation intensity $\left(I_{1,0}(a) / C\right)(24)$ with an increase in the mean QD radius $a$ significantly decreases to a value $3 \cdot 10^{-2}$ at $a=1.5 \mathrm{~nm}$ (see Table 1 ).

When deriving formula $\left(I_{1,0}(a) / C\right)(24)$, the possibility of electron penetration was not taken into account, which was located at the energy level $E_{1,0}{ }^{(e)}(a)(11)$ in the conduction band of the silicon matrix, into the bandgap of the germanium QD (see Figure 1). The ratios of the energies $E_{1,0}{ }^{(e)}(a)(11)$ of the ground state of the electron to the height $\Delta E_{c(S i)}=340 \mathrm{meV}$ of the potential barrier are small, from 0.2 to 0.25 (see Table 1 and Figure 1). At such small values $\left(E_{1,0}{ }^{(e)}(a) / \Delta E_{c(S i)}\right)$, apparently, one can ignore the possibility of electron penetration from the conduction band of the silicon matrix into the bandgap of germanium QDs.

The observed broad band of low-temperature photoluminescence in the experimental work [2] in the energy range (720-820 $\mathrm{meV}$ ) can be caused by the transition of an electron in real space between energy levels (11) and ((15)-(18)) in germanium/silicon heterostructures, containing QDs with mean radii $a$ not exceeding $1.5 \mathrm{~nm}$. The energy of such transitions $\hbar \omega_{e h}(a)(22)$ lies in the interval,

$$
736.4 \mathrm{meV} \leq \hbar \omega_{e h}(a) \leq 1074.2 \mathrm{meV}
$$

in a nanosystem containing germanium QDs with an mean radius $a$ in the range (12) (see Table 1). In this case, the transition energies $\hbar \omega_{e h}(a)$ (27) contain a part of the experimental values of the energies located in the broad bands of low-temperature photoluminescence, in the intervals 720-820 meV.

\section{Discussion}

It is shown that by varying the parameters of germanium/silicon heterostructures with germanium QDs (radii $a$ of QDs, values of effective masses of electrons and holes, depth $\Delta V$ of the potential well for holes in QDs), one can directionally vary the positions 
of the energy levels of electrons and holes, energies of electronic transitions, the possibility of a hole leaving the germanium QD into the bandgap of the silicon matrix, as well as the values of the intensity of radiative recombination caused by the electronic transitions in real space. The latter circumstance, as well as the effect of a significant increase (by more than an order of magnitude) in the intensity of radiative recombination in the nanosystem, opens up new opportunities in the development of fundamental and applied foundations, allowing the creation of a new generation of efficient light-emitting and photodetectors based on germanium/silicon heterostructures with germanium QDs.

\section{Conclusions}

It is shown that as a result of a hole leaving the germanium QD into the bandgap of the silicon matrix, there is a direct transition in real space between an electron located in the conduction band of the silicon matrix and the hole located in the bandgap of the silicon matrix. It is found that as a result of such a direct electronic transition in real space, the intensity of radiative recombination in the germanium/silicon nanosystem with germanium QD increases significantly.

Author Contributions: S.I.P. wrote the Sections 2-4. L.J. wrote the Sections 1, 5 and 6. All authors have read and agreed to the published version of the manuscript.

Funding: This research received no external funding.

Institutional Review Board Statement: Not applicable.

Informed Consent Statement: Not applicable.

Data Availability Statement: Not applicable.

Conflicts of Interest: The authors declare that they have no competing interests.

\section{References}

1. Yakimov, A.I.; Bloshkin, A.A.; Dvurechenskii, A.V. Excitons in Ge/Si double quantum dots. JETP Lett. 2009, 90, 569-573. [CrossRef]

2. Zinovieva, A.F.; Zinovyev, V.A.; Nikiforov, A.I.; Timofeev, V.A.; Mudryi, A.V.; Nenashev, A.V.; Dvurechenskii, A.V. Photoluminescence enhancement in double Ge/Si quantum dot structures. JETP Lett. 2016, 104, 823-826. [CrossRef]

3. Krasilnik, Z.F.; Novikov, A.V.; Lobanov, D.N.; Kudryavtsev, K.E.; Antonov, A.V.; Obolenskiy, S.V.; Zakharov, N.D.; Werner, P. SiGe nanostructures with self-assembled islands for Si-based optoelectronics. Semicond. Sci. Technol. 2011, 26, 014029-014038. [CrossRef]

4. Zinovieva, A.F.; Nikiforov, A.I.; Timofeev, V.A.; Nenashev, A.V.; Dvurechenskii, A.V.; Kulik, L.V. Electron localization in Ge/Si heterostructures with double quantum dots detected by an electron spin resonance method. Phys. Rev. B 2013, 88, 235308. [CrossRef]

5. Dvurechenskii, A.V.; Zinovyeva, A.F.; Zinov'ev, V.A.; Nenashev, A.V.; Smagina, Z.; Teys, S.; Shklyaev, A.; Erenburg, S.; Trubina, S. Photoluminescence from Ordered Ge/Si Quantum Dot Groups Grown on the Strain-Patterned Substrates. Phys. Status Solidi C 2017, 14, 1700187.

6. Zinovieva, A.F.; Zinovyev, V.A.; Nenashev, A.V.; Teys, S.A.; Dvurechenskii, A.V.; Borodavchenko, O.M.; Zhivulko, V.D.; Mudryi, A.V. Photoluminescence of compact GeSi quantum dot groups with increased probability of finding an electron in Ge. Sci. Rep. 2020, 10, 9308. [CrossRef] [PubMed]

7. Grydlik, M.; Hackl, F.; Groiss, H.; Glaser, M.; Halilovic, A.; Fromherz, T.; Jantsch, W.; Schäffler, F.; Brehm, M. Lasing from Glassy Ge Quantum Dots in Crystalline Si. ACS Photonics 2016, 3, 298-303. [CrossRef]

8. Brehm, M.; Grydlik, M. Site-controlled and advanced epitaxial Ge/Si quantum dots: Fabrication, properties, and applications. Nanotechnology 2017, 28, 392001. [CrossRef]

9. Yakimov, A.I.; Dvurechenskii, A.V.; Nikiforov, A.I. Spatial separation of electrons in $\mathrm{Ge} / \mathrm{Si}(001)$ heterostructures with quantum dots. JETP Lett. 2001, 73, 529-531. [CrossRef]

10. Dvurechenskii, A.V.; Yakimov, A.I. Quantum dot Ge/Si heterostructures. Phys. Uspekhi 2001, 44, 1304-1307. [CrossRef]

11. Dvurechenskii, A.V.; Zinov'ev, V.A.; Smagina, Z.V. Self-organization of an ensemble of Ge nanoclusters upon pulsed ir-radiation with low-energy ions during heteroepitaxy on Si. JETP Lett. 2001, 74, 267-269. [CrossRef]

12. Yakimov, A.I.; Dvurechenskii, A.V.; Stepina, N.P.; Nikiforov, A.I.; Nenashev, A.V. Contribution of the electron-electron interac-tion to the optical properties of dense arrays of $\mathrm{Ge} / \mathrm{Si}$ quantum dots. JETP 2001, 92, 500-513. [CrossRef]

13. Yakimov, A.I.; Stepina, N.P.; Dvurechenskii, A.; Nikiforov, A.I.; Nenashev, A.V. Interband absorption in charged Ge/Si type-II quantum dots. Phys. Rev. B 2001, 63, 045312. [CrossRef] 
14. Dvurechenskii, A.V.; Yakimov, A.I. Type II Ge/Si quantum dots. Semiconductors 2001, 35, 1095-1105. [CrossRef]

15. Yakimov, A.I.; Dvurechenskii, A.; Stepina, N.P.; Nenashev, A.V.; Nikiforov, A.I. Spatially indirect excitons in self-assembled Ge/Si quantum dots. Nanotechnology 2001, 12, 441-446. [CrossRef]

16. Dvurechenskii, A.; Nenashev, A.V.; Yakimov, A.I. Electronic structure of Ge/Si quantum dots. Nanotechnology 2002, 13, 75-80. [CrossRef]

17. Yakimov, A.I.; Dvurechenski1̌, A.V.; Bloshkin, A.A.; Nenashev, A.V. Binding of electron states in multilayer strained Ge/Si heterostructures with type-II quantum dots. JETP Lett. 2006, 83, 156-161. [CrossRef]

18. Yakimov, A.I.; Nikiforov, A.I.; Dvurechenski1̆, A.V. Bonding state of a hole in Ge/Si double quantum dots. JETP Lett. 2007, 86, 478-481. [CrossRef]

19. Yakimov, A.I.; Kirienko, V.V.; Bloshkin, A.A.; Armbrister, V.A.; Dvurechenskii, A.V. Strain-induced localization of elec-trons in layers of the second-type Ge/Si quantum dots. JETP Lett. 2015, 101, 750-753. [CrossRef]

20. Stepina, N.P.; Valkovskii, V.V.; Galperin, Y.M.; Smagina, Z.V.; Dvurechenskii, A. Conductance through chains of Ge/Si quantum dots: Crossover from one-dimensional to quasi-one-dimensional hopping. JETP Lett. 2015, 101, 22-26. [CrossRef]

21. Smagina, Z.V.; Dvurechensky, A.V.; Seleznev, V.A. Linear chains of Ge / Si quantum dots during growth on a structured surface formed ion irradiation. Semiconductors 2015, 49, 749-753. [CrossRef]

22. Zinovyev, V.A.; Zinovieva, A.F.; Kuchinskaya, P.A.; Smagina, Z.V.; Armbrister, V.A.; Dvurechenskii, A.V.; Borodavchenko, O.M.; Zhivulko, V.D.; Mudryi, A.V. Strain-induced improvement of photoluminescence from the groups of laterally ordered SiGe quantum dots. Appl. Phys. Lett. 2017, 110, 102101. [CrossRef]

23. Pokutnyi, S.I. On an exciton with a spatially separated electron and hole in quasi-zero-dimensional semiconductor nanosys-tems. Semiconductors 2013, 47, 791-798. [CrossRef]

24. Pokutnyi, S.I. Excitons based on spatially separated electrons and holes in Ge/Si heterostructures with germanium quantum dots. Low Temp. Phys. 2016, 42, 1151-1154. [CrossRef]

25. Pokutnyi, S.I. Exciton spectroscopy with spatially separated electron and hole in Ge/Si heterostructure with germanium quan-tum dots. Low Temp. Phys. 2018, 44, 819-823. [CrossRef]

26. Pokutnyi, S.I. Polarizability of germanium quantum dots with spatially separated electrons and holes. Eur. Phys. J. Plus 2020, 135, 74. [CrossRef]

27. Pokutnyi, S.I. The splitting of electron states in Ge/Si nanosystem with germanium quantum dots. Phys. B Condens. Matter 2021, 601, 412583. [CrossRef]

28. Nenashev, A.V.; Dvurechenskii, A.V. Variational method of energy level calculation in pyramidal quantum dots. J. Appl. Phys. 2020, 127, 154301. [CrossRef]

29. Pokutnyi, S.I. Spectrum of exciton in quasi-zero-dimensional systems: Theory. Phys. Lett. A 1995, 203, 388-392. [CrossRef]

30. Efros, A.L.; Efros, A.L. Interband Absorption of Light in a Semiconductor Sphere. Sov. Phys.-Semicond. 1982, 16, $772-775$. 